\title{
JURISPRUDENCIA AMBIENTAL EN CASTILLA Y LEÓN (PRIMER SEMESTRE 2019)
}

\author{
IÑIGO SANZ RUBIALES \\ Catedrático de Derecho Administrativo \\ Universidad de Valladolid
}


Sumario: 1. Introducción: algunos aspectos (críticos) sobre los Autos de suspensión de la caza en Castilla y León. 2. El auto de suspensión del Decreto de especies cinegéticas. 2.1. Los daños y perjuicios de difícil o imposible reparación. 2.2. La ponderación de intereses. 2.3. La peculiar interpretación del "fumus boni iuris": la sorprendente e injustificada deslegitimación judicial de los informes de la Administración competente y de expertos biólogos aportados por la Junta. 3. El Auto de suspensión de la Orden de caza 2019-2020. 4. Conclusiones. 5. Otras decisiones judiciales.

\section{INTRODUCCIÓN: ALGUNOS ASPECTOS (CRÍTICOS) SOBRE LOS AUTOS DE SUSPENSIÓN DE LA CAZA EN CASTILLA Y LEÓN}

Este comentario, ni mucho menos exhaustivo, se centra en el primero de los dos Autos dictados por la Sala de lo contencioso del Tribunal Superior de Justicia de Castilla y León en fechas 21 y 26 de febrero de 2019, que suspenden el Decreto de especies cinegéticas y la Orden de caza 2019-2020, respectivamente. La razón de ser de este estudio casi monográfico es la extraordinaria trascendencia social, económica y política que han tenido sendos autos: se han multiplicado las noticias de radio, prensa, televisión e internet y todo ello en pre-campaña electoral. $Y$ no solo eso: han dado lugar a una actuación sin apenas precedentes de las Cortes de Castilla y León, que aprobó por vía de urgencia sin ningún voto en contra la reforma de la Ley de Caza para neutralizar el efecto de los autos y permitir el ejercicio de la caza desde los primeros días de abril.

Ahora bien, como tendremos ocasión de comprobar, más que el efecto -ya neutralizado- de impedir la caza en una región con una importante tradición cinegética (en 2018, solo de caza se expidieron 88.992 licencias, según la Agencia ICAL), tiene interés la argumentación utilizada por la Sala en el primer Auto, cuestionada por un contundente voto particular (de idéntica extensión y mayor motivación que el propio Auto) y parcialmente reiterada en el segundo.

\section{EL AUTO DE SUSPENSIÓN DEL DECRETO DE ESPECIES CINEGÉTICAS}


No es nuevo el cuestionamiento en vía judicial de la regulación de especies cinegéticas y de la orden de caza: tenemos precedentes claros de los últimos años. Así, la STSJ de 28 de febrero de 2012 había anulado la Orden 928/2010, anual de caza; posteriormente, la STSJ Castilla y León (Valladolid) de 17 de mayo de 2017 (Rec. 615/2015) anuló algunos preceptos del el Decreto 32/2015, de 30 de abril, de la Junta de Castilla y León, por el que se regula la conservación de las especies cinegéticas de Castilla y León, consecuencia de la cual la Junta aprobó el Decreto 10/2018, de 26 de abril, por el que se modifica el Decreto 32/2015, de 30 de abril. Y el año pasado también anuló la Orden FYM/609/2016, de 28 de junio, por la que se aprueba la Orden Anual de Caza de 2016 que, no obstante, ya había sido derogada por la Orden FYM/528/2017, de 27 de junio.

De todas las actuaciones anuladas tiene especial trascendencia el Decreto de conservación de especies cinegéticas porque a él se va a referir la Sala en varias ocasiones para justificar el fallo.

El Partido Animalista (PACMA) había recurrido el Decreto 10/2018, de 26 de abril, de modificación del Decreto 32/2015, de 30 de abril (consecuencia de la anulación de algunos de sus preceptos por la STSJ de 17 de mayo de 2017) y relativo a la conservación y aprovechamiento de las especies cinegéticas de Castilla y León, que se desarrolla anualmente mediante las órdenes de caza, y por medio de otrosí en la demanda solicitó su suspensión. Según la normativa regional, especies cinegéticas son aquellas susceptibles de aprovechamiento cinegético en abstracto, de acuerdo con una serie de criterios (art. 13 Decreto); cada orden anual de caza concreta cuáles de las especies cinegéticas (en abstracto) van a poder ser cazables (en concreto) esa temporada (art. 14 Decreto). La alegación del recurrente para pedir la suspensión era que la aplicación de la norma impugnada "va a producir daños de imposible o difícil reparación, ya que se va a autorizar la actividad cinegética de determinadas especies silvestres sin contar con los estudios científicos precisos que lo avalan" (FD 1으. 
La Sala recuerda (FD $4^{\circ}$ ) que el principal criterio para la adopción de las medidas cautelares, cualquiera que sea la que se interese, es que la ejecución del acto o la aplicación de la disposición recurrida pueda hacer perder al recurso su finalidad legitima, pero siempre sobre la base de una ponderación suficientemente motivada de todos los intereses en conflicto. Pero utiliza para ello el criterio del "fumus boni iuris", no recogido por la LJCA y admitido con muchas restricciones por la jurisprudencia, por el riesgo evidente de que puede propiciar una resolución anticipada del fondo del asunto. Según el art. 130 LJCA,

“1. Previa valoración circunstanciada de todos los intereses en conflicto, la medida cautelar podrá acordarse únicamente cuando la ejecución del acto o la aplicación de la disposición pudieran hacer perder su finalidad legítima al recurso.

2. La medida cautelar podrá denegarse cuando de ésta pudiera seguirse perturbación grave de los intereses generales o de tercero que el Juez o Tribunal ponderará en forma circunstanciada".

Esta previsión debe completarse con lo establecido por el art. 133.1:

"Cuando de la medida cautelar pudieran derivarse perjuicios de cualquier naturaleza, podrán acordarse las medidas que sean adecuadas para evitar o paliar dichos perjuicios".

Es preciso, por tanto, ponderar los intereses en conflicto y considerar si la adopción de la medida cautelar sirve o no para asegurar la finalidad del recurso.

\subsection{Los daños y perjuicios de difícil o imposible reparación}

a) La inversión de la carga de la prueba de los daños y perjuicios de difícil o imposible reparación 
El recurrente alega que la aplicación de la norma impugnada va a producir "daños de imposible o difícil reparación, ya que se va a autorizar la actividad cinegética de determinadas especies silvestres sin contar con los estudios" (FD $\left.1^{\circ}\right)$. Sorprendentemente, la Sala no exige que se acrediten tales daños; es decir, los da por acreditados (i!) (FD 7º) y cuestiona, sin embargo, la alegación de la Administración. En efecto, es el propio Tribunal el que invierte la carga de la prueba, al establecer -con cita de las Directivas hábitats- que el hecho de que "las especies a las que se refiere el Decreto se encuentren dentro de los citados Anexos [de la Directiva: susceptibles de caza] no implica que la ejecución de la norma impugnada no cause daños irreparables o de difícil reparación, porque la práctica de la actividad cinegética y las medidas de gestión que se contemplen exigen contar con información relativa al nivel de población".

Por tanto, el Auto invierte la carga de la prueba; no exige que se acrediten los daños y perjuicios de difícil o imposible reparación porque va de suyo: parte de la base de que la información aportada por la Administración pública no permite asegurar la inocuidad del ejercicio de la caza.

Pone el dedo en la llaga el voto particular, cuando recuerda $\left(2^{\circ}\right)$ : "(...) lo que no puede es invertirse el principio de carga de la prueba y partir de la consideración preconcebida de que en cuanto que no se ha justificado el estado de conservación de cada una de las especies -cuestión aún no analizada y que solo se puede hacer desde la óptica propia de esta "sumaria cognitio"-, de ello mismo deriva la irreversibilidad del perjuicio causado. Antes al contrario, el perjuicio debe concretarse en cada caso por la parte actora y nada encontramos sobre ello...".

b) Mantener el vigor de una norma de declaración de especies cinegéticas no causa directamente daños, ni reparables ni irreparables.

El Tribunal olvida que el Decreto de especies cinegéticas no tiene aplicación directa: solo pueden ser objeto de caza las especies (cinegéticas) incluidas en la Orden anual de caza, de acuerdo con las peculiares circunstancias 
coyunturales (anuales) de la especie (arts. 13 y 14 del Decreto). Es la orden de caza la que debe determinar, a la luz de las circunstancias en juego, si una temporada es cazable o no alguna de las especies cinegéticas. $Y$ no hay que olvidar que ambas disposiciones -Decreto y Orden de caza- estaban impugnadas a la vez.

Por otra parte, a la hora de valorar los perjuicios derivados de la vigencia del Decreto, la Sala debería haber tenido en cuenta todas las demás técnicas de protección de las especies que incluye la Ley y que contribuirían -en caso de que se hubiese mantenido la vigencia del Decreto- a limitar los potenciales perjuicios a dichas especies: la prohibición de caza fuera de terrenos cinegéticos (art. 19.2), la obligatoria aprobación de planes cinegéticos (que incluyen la situación poblacional y la cuantía de las capturas estimadas: art. 40), las órdenes de caza, que deben incluir las regulaciones y las épocas hábiles de caza aplicables a las distintas especies en las diversas zonas, con expresión de las distintas modalidades y capturas permitidas, de acuerdo con los informes de los Consejos territoriales y del órgano central colegiado competente para ello (antiguo art. 41), las otras limitaciones al ejercicio de la caza (arts. 42 y ss.), etc..

c) La prohibición inmediata y total de la caza puede romper el equilibrio ecosistémico aunque beneficie a algunas especies. Olvido de los efectos del ejercicio de la caza en los años anteriores

La prohibición de la caza en su totalidad de un año para otro supone eliminar, sin periodo transitorio alguno, uno de los elementos de presión sobre la fauna cinegética, co-responsable en muy alta medida del equilibrio ecosistémico de la biodiversidad. La eliminación total de dicha presión con eficacia inmediata, si bien puede beneficiar a algunas especies, no se lleva a cabo mediante un periodo transitorio que permita mantener el equilibrio del ecosistema mediante la retirada progresiva del ejercicio de la caza, por lo que puede generar un desequilibrio notable. 
La presunción del Tribunal de que la suspensión de la caza va a evitar daños y perjuicios irreparables no tiene apoyo fáctico ni jurídico alguno. Resulta ingenuo creer que la prohibición de caza de todas las especies de un año para otro sea positiva en todo caso. Será positiva (cuantitativamente hablando) para algunas especies y en algunos casos y será negativa para el equilibrio del ecosistemas en otros casos: así, el excesivo número de ungulados silvestres afecta a los recursos tróficos de un ecosistema; asimismo, numerosas especies -conejos, p. ej.- que tienen una extraordinaria capacidad reproductiva y cuentan con un elevado número de ejemplares, sin la presión de la caza se pueden convertir en plaga... y eso no es bueno para el equilibrio ecosistémico.

d) Frente al planteamiento del Auto - la caza como actividad contraria al medio ambiente- hay que pasar a conceptuar la caza como actividad promotora de servicios ambientales

El Auto parte del prejuicio de que la caza de especies cinegéticas es contraria al medio ambiente (por tanto, de que los cazadores son, en cuanto tales, agresores del ambiente). Se trata de un planteamiento simplista y urbanita, que choca con la experiencia de muchos. No me resisto a dejar de citar a Miguel Delibes, que se definía a si mismo de la siguiente manera, justificando la compatibilidad entre caza y ambiente: "No soy un escritor que caza, sino un cazador que escribe... Soy un ecologista que escribe y caza". Es cierto que la caza incontrolada puede dañar el ambiente, como lo puede hacer industria, el transporte y la actividad doméstica si no tienen controles ad hoc. Pero la caza controlada, de especies especialmente elegidas, de acuerdo con la cantidad anual de piezas, en cotos planificados, etc... no es dañina para el medio ambiente. Los cazadores complementan el servicio ambiental -dirigido al equilibrio ecosistémico y al aprovechamiento económico y lúdico-propio de los depredadores en un territorio fuertemente humanizado que ha ido progresivamente ocupando los territorios de campeo de caza de dichos depredadores.

\subsection{La ponderación de intereses}


El Auto alude -en aplicación del art. 130 LJCA- a la ponderación de intereses para determinar la adopción de la medida cautelar. Aquí se plantean dos problemas:

a) por una parte, el Auto crea una contraposición de intereses que no existe con el carácter absoluto que afirma. Según él, deben valorarse los intereses de las especies (FD $7^{\circ}$ ) frente a los económicos y sociales; este planteamiento no es real, porque la ponderación debe plantearse entre la protección de las especies, efectivamente, y la protección del medio ambiente y de los intereses económicos y sociales. O dicho de otra manera, debemos hablar del mantenimiento y crecimiento numérico de las especies cinegéticas vs. equilibrio ecológico ambiental. En efecto, hace ya mucho tiempo que dejó de entenderse la protección de la biodiversidad en términos meramente cuantitativos: actualmente, se trata, no de que haya un elevado número de individuos de las especies silvestres (si fuese así, las plagas serían buenas) sino del mantenimiento de los servicios ambientales. $Y$ los servicios ambientales de la biodiversidad derivan del equilibrio del ecosistema: por lo tanto, lo que debe buscar el Derecho ambiental (y los jueces) es el equilibrio y los servicios, no el número absoluto de individuos.

b) El segundo problema es que el Auto evita toda ponderación: señala apodícticamente que el interés de las especies cinegéticas prima sobre los intereses económicos y sociales, pero no explica por qué. O lo resuelve con una valoración subjetiva sobre la reparabilidad (FD $8^{\circ}$ ): "no desconocemos que, igualmente, como pone de manifiesto la Administración demandada, la suspensión de esta norma puede producir daños a los intereses generales y de terceros, y así se pone de manifiesto en el informe que acompaña a su escrito de oposición, pero entendemos que en una ponderación de todos los intereses en conflicto, el interés más sensible, más frágil y vulnerable y, por ello, más necesitado de protección es el de la conservación de la fauna silvestre" [cinegética, se entiende]. Y sigue diciendo: "los daños para los intereses generales y de terceros a los que hace referencia el informe que se 
aporta por la Administración demandada están expuestos en términos genéricos $y$, desde luego, son de más fácil reparación". También el voto particular sale al paso de esta afirmación cuasi-axiomática: "en todo caso, no puede compartirse como afirmación apodíctica que la conservación de la fauna silvestre es el interés más necesitado de protección, pues dichos intereses necesitados de protección se han de deducir del ordenamiento (...)".

$\mathrm{Ni}$ que decir tiene que en cuatro líneas no pueden ponderarse mínimamente los intereses en juego, y menos declarar sin justificación alguna la resiliencia de cada uno de los recursos dañados. No tiene en cuenta la capacidad de recuperación en términos cuantitativos de una buena parte (quizá la mayoría) de las especies cinegéticas, en un periodo de tiempo corto. Pero, en todo caso, para una decisión de este calibre, con estos efectos, falta la "valoración circunstanciada de todos los intereses en conflicto" que exige el art. 130 LJCA y que el Tribunal, de forma evidente, no hace. Adolece, por tanto, de un déficit efectivo de motivación (lo mismo de lo que acusa a la Administración autonómica).

\subsection{La peculiar interpretación del "fumus boni iuris": la sorprendente e injustificada deslegitimación judicial de los informes de la Administración competente y de expertos biólogos aportados por la Junta.}

La Administración autonómica, utilizando la fórmula del fumus boni iuris, pasa a cuestionar los informes utilizados por la Junta de Castilla y León para justificar el contenido del Decreto de especies cinegéticas sustitutivo del anulado por la Sala por sentencia de 17 de mayo de 2017. Porque "efectivamente, las probabilidades de que la ejecución del Decreto cause daños irreparables en la fauna silvestre están en función de los estudios científicos realizados para declarar una especie como cinegética".

$\mathrm{Y}$, sin entrar a valorarlos en su contenido, los califica como no-actualizados y no objetivos, consecuencia de lo cual confirma el "fumus boni iuris" de la petición de suspensión. 
Respecto de la falta de actualización, dice la sentencia:

"Tal y como se expone en su introducción, para determinar el nivel poblacional de cada especie se han consultado las guías regionales sobre aves y mamíferos de los años 2001 y 2005 así como lo que denomina "sistema de censo, seguimiento y estudio de tendencias de las especies cinegéticas en Castilla y León". Dicho sistema está compuesto por información resultante de determinadas bases de datos que recogen los aprovechamientos cinegéticos de cada especie a lo largo de las temporadas de caza (2001 hasta el 2017), COGE, también del banco de datos de la actividad cinegética en Castilla y León, CAZDATA (temporadas 1991 a 2017) y otros programas llevados a cabo por SEO/BirdLife (programa SACRE, programa SACIN) y de seguimiento de aves acuáticas. También se ha utilizado el sistema de seguimiento de algunos animales de fauna silvestre realizados por los Agentes Medioambientales y Celadores de Medio Ambiente de la Junta de Castilla y León, así como la información de la propia actividad administrativa en materia de caza".

Pero lo mejor es la conclusión: "pues bien, una lectura de dichas fuentes pone de manifiesto que no son actuales, ya que se toman en consideración datos de los años 2001 y 2005“. Es decir, el Auto deslegitima toda la información aportada por la Junta, porque tiene en cuenta (entre otros), datos de 2001 y 2005. ¿Pero no reconoce el propio Auto -y el autor del informe- que el informe se ha realizado utilizando bases de datos que llegan hasta 2017? Por otra parte, hay que recordar que se está analizando un Decreto -de especies cinegéticas- con tendencia a mantenerse en el tiempo, y no una mera orden de caza de vigencia anual. Esto supone que el Decreto debe elaborarse a la luz del análisis de las pautas de evolución cuantitativa y distributiva de las especies a lo largo del tiempo, sin perjuicio de que las órdenes anuales de caza posibiliten, limiten o no prevean en determinados años la actividad cinegética sobre alguna especie. 
El voto particular muestra una especial contundencia ante el evidente voluntarismo de la valoración de la Sala, y trae a colación el informe del Jefe de Servicio de Caza y Pesca, que señala: "teniendo en cuenta tanto los datos de que dispone la Administración del seguimiento continuado de las mismas [especies cinegéticas], que forman parte del expediente administrativo del Decreto impugnado, así como los datos de capturas comunicados por los titulares cinegéticos, es posible afirmar que en las últimas temporadas cinegéticas se han mantenido o incluso, en muchos casos, incrementado sus niveles poblacionales en la Comunidad de Castilla y León (...). El estado de conservación de estas especies no se verá alterado como consecuencia de la práctica de la caza. Se considera, por el contrario que "la actividad cinegética se erige en el procedimiento idóneo y adecuado para contribuir también a su propia sostenibilidad".

Pero el Magistrado discrepante apunta más indicios de la legitimidad de los estudios e informes encargados y aportados por la Administración y no contradecidos por informe alguno de la parte contraria. El director del equipo que elaboró el informe señaló: "[no solo] es falso en la disciplina que nos ocupa sino que sería iluso que en cualquier país del mundo hubiera una información anual, bienal, trianual ... para cada especie y para cada uno de los aspectos bioecológicos que dinamizan los ciclos biológicos. Pero es que encima ya hemos visto, buena parte de la información analizada llega incluso hasta 2017".

En definitiva, el experto profesional biólogo muestra cómo los informes elaborados y aportados por su equipo se sitúan dentro de lo exigible por el principio de proporcionalidad aplicado a su actividad técnica, en la medida en que los que los estudios anuales, bianuales o trianuales pormenorizados y cuantificados sobre la evolución de las especies se refieren exclusivamente a algunas de las especies especialmente protegidas (oso pardo, lobo ibérico, lince, águila imperial), ni siquiera a todas las protegidas, y por supuesto, en ningún caso a todas las cinegéticas. Ad impossibilia nemo tenetur.

Es sorprendente, por ello, que, sin un solo informe técnico que desdiga lo afirmado por el responsable, y sin conocimiento de la lex artis de los estudios 
generales sobre especies cinegéticas, la Sala (formada por juristas, no por biólogos) deslegitime de plano este y los demás informes. Da la impresión de que la Sala está sustituyendo a la Administración en el campo de su discrecionalidad técnica.

Respecto del carácter "no objetivo" de la información, dice a continuación: "Por otro lado, no consta que se hayan utilizado estudios científicos objetivos, ya que el llamado "sistema de censo, seguimiento y estudio de tendencias de las especies cinegéticas en Castilla y León" se nutre de una información recopilada para otros fines, y no específicamente para los fines a los que ha de servir el Decreto". Es decir, para la Sala, la falta de objetividad no deriva del contenido de la información (que no se ajuste a la realidad objetiva) sino a que se recopila para otros fines (i!). Sin comentarios.

\section{EL AUTO DE SUSPENSIÓN DE LA ORDEN DE CAZA 2019-2020}

Cuatro días después del Auto suspensivo del Decreto de especies cinegéticas, la misma Sala suspendió igualmente la Orden FYH/728/2018, de 25 de junio, de caza para la temporada 2019-2020. En este caso se reitera el voto particular del mismo Magistrado discrepante en el primer Auto, aunque la decisión judicial es reglada y meramente declarativa: si la norma en la que se apoya la Orden está suspendida, no puede ejecutarse la Orden; sin la determinación de las especies cinegéticas en abstracto, no puede haber caza en concreto (y así lo confirma el Auto en el FD 6ํำ in fine).

En este Auto reconoce la Sala algo que no mencionó ni aplicó en el primero: la necesidad de una especial ponderación de circunstancias y la necesidad de justificar los daños y perjuicios irreparables derivados de la ejecución del acto por parte del solicitante de la suspensión (FD $3^{\circ}$ in fine): señala, en efecto, que "la decisión sobre la procedencia de la medida cautelar comporta un alto grado de ponderación conjunta de circunstancias por parte del Tribunal, que requiere la necesidad de justificación y prueba de aquellas que pueden permitir efectuar la valoración de la procedencia de la medida, correspondiendo al interesado la 
carga de probar qué daños y perjuicios de reparación imposible o difícil concurren en el caso para acordar la medida cautelar que solicita".

Como novedad, incluye una justificación (inexistente en el primero) del carácter irreparable de los daños a las especies cinegéticas si se ejecutase la orden (FD $5^{\circ}$ ): "estos daños, de producirse, han de ser calificados de irreparables o de difícil reparación, que es el planteamiento que hace la parte actora, puesto que los ejemplares cazados no son recuperables". Estamos ante un mensaje sorprendente, ajeno a lo jurídico-ambiental, basado en la confusión entre derecho ambiental (cuyo objeto es la protección de las especies y sus hábitats) y ética animal (dirigida a la protección del bienestar individualizado de los animales). Por lo pronto, deslegitima la posibilidad de cazar, porque toda pieza cazada y muerta es, per se, irrecuperable y, por tanto, irreparable; por otra parte, deja sin sentido la normativa de responsabilidad medioambiental, que se dirige a reparar los daños a la biodiversidad, y todo daño a la biodiversidad conlleva, de ordinario, la muerte de ejemplares individuales animales 0 vegetales; ¿no se puede reparar la pérdida de ejemplares con sueltas (o con plantaciones, si son vegetales)? ¿Y las sueltas de perdices, de conejos, de ciervos..., de truchas? ¿Y las reforestaciones? ¿y los bancos de biodiversidad? Indudablemente, la argumentación de la Sala sí que es aplicable a especies en situación crítica, esto es, en peligro de extinción o incluso en riesgo de estarlo (vulnerables), porque en ocasiones la muerte de algún individuo de estas puede llegar a ser, efectivamente, irreparable (como ocurrió con el último bucardo pirenaico, en cuanto afecta al servicio ambiental que presta la especie); pero no resulta aplicable a las especies que no están incluidas en el Listado de Especies Silvestres en Régimen de Protección Especial y que, por ello, son susceptibles de ser declaradas cinegéticas (en abstracto).

\section{CONCLUSIONES}

No entra este comentario en aspectos técnicos propios del orden contenciosoadministrativo, como la supuesta invalidez del informe del Consejo del Consejo Regional del Medio Ambiente, la utilización del "fumus boni iuris" o la posible 
incongruencia "extra petitum", entre otros, porque se salen de lo estrictamente ambiental, aunque lo haga, críticamente, el Voto particular.

Se trata, como hemos visto, de un Auto (el de 21 de febrero) voluntarista, sin motivación suficiente, sin ponderación efectiva de intereses, que yerra en la contraposición de los intereses en juego y que posiblemente esté sustituyendo a la Administración en la valoración técnica de la exclusiva competencia administrativa.

Con todos sus defectos, estos Autos han tenido una enorme virtud, y ha sido la de poner de acuerdo a la práctica totalidad de los representantes populares en las Cortes de Castilla y León, que se han apresurado a aprobar una modificación de la Ley de Caza que neutralice los daños que podrían derivarse del Auto. Y todo ello, con el voto a favor de todos los procuradores presentes en el hemiciclo.

\section{OTRAS DECISIONES JUDICIALES}

Al margen de los Autos de suspensión de los diversos instrumentos regulatorios de la caza, el Tribunal Superior de Justicia ha proseguido su actividad ordinaria relativa a la protección de la legalidad ambiental. Merece la pena destacar, por contraste con los Autos arriba citados, la STSJCYL (Valladolid) de 7 de noviembre de 2018, que resuelve la impugnación del Acuerdo 78/2016, de 1 de diciembre, de la Junta de Castilla y León, por el que se determinan actuaciones fitosanitarias de lucha contra el desarrollo de las poblaciones de topillo campesino en Castilla y León. El recurso se basa en la falta de justificación de la existencia de plaga y, fundamentalmente, en la autorización de uso de rodenticidas, que implican un "inasumible riesgo ambiental", según el recurso. La Sala confirma la suficiente motivación de la declaración, a la luz de la documentación aportada por la Junta (acta de la comisión de expertos, el acta de comisión de roedores, y el Informe de Evolución y estado de situación, entre otros); respecto de los rodenticidas, la Sala reconoce que su uso -excepcional y sometido a fuertes limitaciones- está autorizado por el Ministerio, por lo que no puede entenderse ilegal (si se utiliza 
según las condiciones previstas). Pero sí que anula, con razón, la posibilidad de quemar rastrojos como técnica de lucha contra el topillo, porque no es una de las medidas previstas en el art. 5 del RD 409/2008, de 28 de Marzo, por el que se establece el programa nacional del control de plagas del topillo campesino, y porque no se justifica, tampoco, los motivos de su utilización en dicho acuerdo.

Finalmente, ya en el ámbito de la tutela de las aguas, cabe destacar la STSJCYL (Burgos), de 20 de diciembre de 2018, que anula la denegación operada por la Confederación del Tajo de la solicitud de autorización planteada por el Ayuntamiento de Navaluenga para la instalación de mercadillo semanal y estructuras para ferias, exposiciones y atracciones, durante los meses de julio, agosto y septiembre, en una parcela ubicada en un tramo clasificado como Área de Riesgo Potencial Significativo de Inundación. La denegación se justificó en razones de seguridad, pero la Sala entendió aplicable el artículo 9 ter) del RDPH, introducido a través de la reforma llevada a cabo por el RD $638 / 2016$, referido específicamente a obras y construcciones en la zona de flujo preferente en suelos en situación básica de suelo urbanizado, que permite realizar nuevas edificaciones $u$ obras de reparación o rehabilitación que supongan un incremento de la ocupación. 